Check for updates

Cite this: Phys. Chem. Chem. Phys. 2018, 20, 21043

Received 7th June 2018

Accepted 25th July 2018

DOI: $10.1039 / c 8 c p 03594 f$

rsc.li/pccp

\section{The effect of strain and functionalization on the optical properties of borophene $\dagger$}

\author{
A. Mogulkoc, (D)*a Y. Mogulkoc, (D) ${ }^{b}$ D. Kecik ${ }^{c d}$ and E. Durgun (D) ${ }^{c}$
}

Following its synthesis, borophene has drawn noticeable attention due to its remarkable intrinsic properties. Understanding and modifying these properties are crucial for implementation of borophene in hightechnological applications. In this study, we employed ab initio techniques to examine the variation of the optoelectronic properties of buckled borophene by strain and surface functionalization. We find that the optical response can be tuned by applying compressive/tensile strain and covering the surface with hydrogen and fluorine atoms. It is shown that the variations in optical properties can be correlated with structural deformations and modifications in the electronic band structure. Revealing the tunability of the optical response of borophene can pave the way for its potential uses in various optoelectronic devices.

\section{Introduction}

Two-dimensional (2D) materials exhibit exceptional electronic and optical properties distinctively different from their bulk counterparts, as a consequence of their reduced dimension (i.e., quantum confinement in the direction perpendicular to the $2 \mathrm{D}$ plane). ${ }^{1}$ Many $2 \mathrm{D}$ systems interact strongly with light over a wide range of the electromagnetic spectrum which allows them to be used in various optoelectronic applications. ${ }^{1-5}$ Graphene, which is a semi-metal with linear band dispersion near the Dirac points, ${ }^{6}$ displays a significant optical response from infrared to ultraviolet wavelengths, making it suitable for interesting applications in nanophotonics and nanoelectronics. ${ }^{7,8}$ On the other hand, metallicity and low optical absorption in the early frequency range are limiting factors for graphene based devices. ${ }^{9,10}$ In contrast, monolayer transition metal dichalcogenides (TMDC) in the form $\mathrm{MX}_{2}\left(\right.$ e.g., $\left.\mathrm{MoS}_{2}\right)$ are direct band gap semiconductors with a range of $\sim 1.0-2.5 \mathrm{eV}^{11}$ and can be used in light emitting diodes (LEDs) ${ }^{12}$ and photodetectors ${ }^{13}$ where emission is dominated by excitons and trions. ${ }^{14}$ Interestingly, a recently realized 2D allotrope of black phosphorus (phosphorene) has a high-mobility and layer-dependent direct band gap of

\footnotetext{
${ }^{a}$ Department of Physics, Faculty of Sciences, Ankara University, Ankara, 06100, Turkey.E-mail: mogulkoc@science.ankara.edu.tr

${ }^{b}$ Department of Physics Engineering, Faculty of Engineering, Ankara University, Ankara, 06100, Turkey

${ }^{c}$ UNAM - National Nanotechnology Research Center and Institute of Materials Science and Nanotechnology, Bilkent University, Ankara, 06800, Turkey.

E-mail: kecik@unam.bilkent.edu.tr, durgun@unam.bilkent.edu.tr

${ }^{d}$ Department of Physics, Bilkent University, Ankara, 06800, Turkey

$\dagger$ Electronic supplementary information (ESI) available: The optical response of the system along transverse and out-of-plane directions, and the variation of buckling length with strain. See DOI: 10.1039/c8cp03594f
}

0.3-2.0 eV which bridges metallic and large band gap systems, thus extending the possibilities in nanophotonics and nanoelectronics. ${ }^{3,15,16}$ Additionally, phosphorene has a puckered geometry which induces in-plane anisotropy to the optoelectronic response of the system. ${ }^{15,17}$

The synthesis of atomically thin boron sheets (i.e., borophene) on silver substrates extended the family of monoelemental 2D materials to group III elements. ${ }^{18}$ The aforementioned polymorph of borophene ( $\delta_{6}$-borophene) has a close-packed, buckled triangular structure and exhibits metallic character different from bulk boron allotropes. ${ }^{18}$ Owing to its out-of-plane buckling, it displays unique and anisotropic electronic, ${ }^{18,19}$ mechanical, ${ }^{18,20}$ and thermal properties. ${ }^{21-23} \delta_{6}$-Borophene is also predicted to possess superconducting behavior ${ }^{24,25}$ at low temperatures which can be tuned by doping and strain. ${ }^{26}$ Following the realization of the buckled phase, the planar geometry of borophene with different arrangements of hexagonal holes $\left(\beta_{12}\right.$ and $\left.\chi_{3}\right)$ has been grown ${ }^{27}$ and various other phases with exceptional properties are foreseen. $^{24,28-31}$ These unique features offer borophene as a promising 2D material for various technological applications. ${ }^{32-34}$

Additionally, borophene also has novel and strongly anisotropic optical properties. Peng et al. ${ }^{19}$ have reported that $\delta_{6}$-borophene exhibits high optical transparency and electrical conductivity along the uncorrugated direction. In a computational study, it has been shown that the oxidation of $\delta_{6}$-borophene significantly increases the low optical conductivity and reflectance of the pristine system especially in the infrared region. ${ }^{35}$ The obtained results also show that the optical response of borophene is prone to surface modifications. Adamska et al. have shown that the optoelectronic properties of borophene are sensitive to applied strain in correlation with charge localization and change in the bond lengths. ${ }^{36}$ Finally, Mortazavi et al. ${ }^{37}$ have examined the optical properties of a planar, hexagonal lattice of 
boron atoms saturated by hydrogen atoms (borophene hydride), which was realized recently ${ }^{38}$ following its theoretical prediction, ${ }^{39}$ and have found that the absorption edge of this material occurs in the visible range, and thus can absorb visible light. Integrating its good metallic character with novel optical properties, borophene can be used for various optoelectronic applications including photovoltaic solar cells, touch screens, and nanodetectors. ${ }^{1}$

Motivated by the recent experimental advances and attractive material properties of borophene polymorphs, we explore the variation of the optoelectronic properties of a close-packed, buckled triangular structure of borophene (which will be referred to simply as borophene) by applying strain and surface functionalization. Firstly, we apply compressive and tensile uniaxial strain between $-8 \%$ to $16 \%$ along the corrugated and uncorrugated directions and examine the modification of the electronic and optical properties. We quantify the transition energies along high symmetry directions with strain and correlate them with significant optical excitations. Next, we saturate the borophene surface with hydrogen and fluorine atoms and characterize the optical response of these systems while comparing them with the pristine structure.

\section{Computational methodology}

The ground-state calculations were performed by using first principles methods within the framework of density functional theory (DFT), ${ }^{40,41}$ implemented in the Vienna ab initio simulation package (VASP). ${ }^{42-45}$ Projector-augmented wave (PAW) potentials ${ }^{46}$ with a plane-wave cutoff energy of $550 \mathrm{eV}$ was used. The exchange-correlation term was approximated by the generalized gradient approximation (GGA) within the Perdew-Burke-Ernzerhof (PBE) functional. ${ }^{47,48}$ The Brillouin zone was sampled with a $24 \times 24 \times 1 k$-point mesh by using the Monkhorst-Pack method. ${ }^{49}$ The convergence criterion for total energy minimization was set to $10^{-8} \mathrm{eV}$ between two sequential steps. Structural optimizations were performed using the conjugate gradient method allowing a maximum $0.001 \mathrm{eV} \AA^{-1}$ force tolerance on each atom. A vacuum space of $20 \AA$ along the non-periodic $z$-direction of the supercell was used to eliminate the interaction between the periodic images.

The random phase approximation (RPA $)^{50-52}$ on top of the PBE approach was adopted to calculate the optical response. A finer $k$-point sampling of $127 \times 127 \times 1$ was used for PBE-RPA calculations including a total number of 96 bands. Local field effects were accounted for, both at the levels of the Hartree and the exchange-correlation potential. The linear response of the system to the light-matter interaction is described by the complex dielectric function, $\varepsilon(\omega)=\varepsilon_{1}(\omega)+\mathbf{i} \varepsilon_{2}(\omega)$, where the Kramers-Kronig relation is applied to obtain the real $\left[\varepsilon_{1}(\omega)\right]$ and imaginary $\left[\varepsilon_{2}(\omega)\right]$ parts. The frequency dependent optical conductivity is given by the relation,

$$
\sigma(\omega)=\frac{\omega}{4 \pi} \operatorname{Im} \varepsilon(\omega)
$$

Both $\varepsilon_{2}(\omega)$ and $\sigma(\omega)$ are calculated for light polarized along $x$ and $y$-directions, in order to describe the in-plane optical response.
Taking into account the metallic nature of borophene, a Drude term was added to $\varepsilon(\omega)$ (both for intraband and interband transitions) by using particular plasma frequency and relaxation time values which were calculated for pristine, strained, and functionalized borophene cases.

\section{Results and discussion}

The optimized atomic structure of pristine borophene is shown in Fig. 1(a). The lattice constants are calculated as $a=2.87 \AA$ and $b=1.62 \AA$ along zigzag (corrugated) and armchair (uncorrugated) directions, respectively, where the buckling height $(\Delta h)$ is found to be $0.90 \AA$, which are all in agreement with previous studies. $^{18,19}$ As illustrated in Fig. 1(b), borophene has an anisotropic metallic character with bands crossing the Fermi level $\left(E_{\mathrm{F}}\right)$ only along the directions parallel to the uncorrugated $(\Gamma-Y$ and $X-S)$ direction. ${ }^{18,19}$ Important interband transitions can be observed rather near and along the $\Gamma-X$ and $S-Y$ directions, where the direct band transition energies from the valence band maximum to the conduction band minimum (d-BTE) at $\Gamma$ and $S$ high-symmetry points are given in Table 1.

\section{A. The effect of strain}

Considering the enhanced stability of borophene under strain ${ }^{19,35}$ and its anisotropic mechanical response, ${ }^{18,20}$ it is essential to examine the effect of strain $(\epsilon)$ on the optoelectronic properties of the system. Earlier it was predicted that tensile strain up to $6 \%$ results in moderate deformations of borophene. ${ }^{20,36}$ While borophene is soft towards deformations along the zigzag direction due to its buckled atomic configuration, it is more difficult to deform it along the armchair direction. Accordingly, the ultimate tensile strength was calculated as $23 \mathrm{GPa} \mathrm{nm}$ at $\epsilon_{y} \approx 0.1$ and $14 \mathrm{GPa} \mathrm{nm}$ at $\epsilon_{x} \approx 0.15 .^{20} \mathrm{In}$ order to reveal the effect of strain on the electronic and optical properties, we apply uniaxial compressive (up to $-8 \%$ ) and tensile strain (up to $16 \%$ ) along corrugated $\left(\epsilon_{x}\right)$ and uncorrugated $\left(\epsilon_{y}\right)$ directions (see Fig. 1(a)). The considered strain range covers weak, moderate, and strong deformations.

The resulting structures under strain are obtained by fixing the lattice constant in the direction of applied load and optimizing

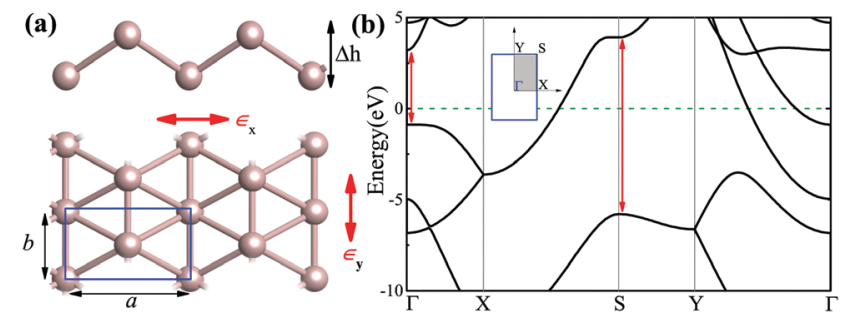

Fig. 1 (a) Atomic configuration and (b) electronic band structure of pristine borophene. High symmetry points along the irreducible Brillouin zone are shown as an inset. The lattice constants along zigzag (a) and armchair (b) directions and the buckling height $(\Delta h)$ are denoted on the unit cell. The directions of the applied strain and examples of two representative direct band widths are shown with red arrows. The Fermi level is set to zero and is shown by the dashed green line. 
Table 1 The variation of direct band transition energies (d-BTE) at high symmetry points $(\Gamma$ and $S)$ with compressive/tensile strain along zigzag $\left(\epsilon_{x}\right)$ and armchair directions $\left(\epsilon_{y}\right)$

\begin{tabular}{|c|c|c|c|c|c|c|c|c|c|c|c|c|c|c|}
\hline \multirow{2}{*}{$\frac{\text { Strain }(\%)}{\text { Symmetry points }}$} & \multicolumn{2}{|l|}{-8} & \multicolumn{2}{|l|}{-4} & \multicolumn{2}{|l|}{0} & \multicolumn{2}{|l|}{4} & \multicolumn{2}{|l|}{8} & \multicolumn{2}{|l|}{12} & \multicolumn{2}{|l|}{16} \\
\hline & $\Gamma$ & $S$ & $\Gamma$ & $S$ & $\Gamma$ & $S$ & $\Gamma$ & $S$ & $\Gamma$ & $S$ & $\Gamma$ & $S$ & $\Gamma$ & $S$ \\
\hline d-BTE $\left[\rightarrow \epsilon_{x}\right](\mathrm{eV})$ & 6.31 & 9.74 & 5.60 & 9.72 & 4.09 & 9.70 & 2.97 & 9.58 & 2.03 & 9.51 & 1.25 & 9.43 & 0.55 & 9.44 \\
\hline $\mathrm{d}-\mathrm{BTE}\left[\rightarrow \epsilon_{y}\right](\mathrm{eV})$ & 4.76 & 11.67 & 4.52 & 10.58 & 4.09 & 9.70 & 3.75 & 8.92 & 3.46 & 8.26 & 1.83 & 8.27 & 0.22 & 8.31 \\
\hline
\end{tabular}

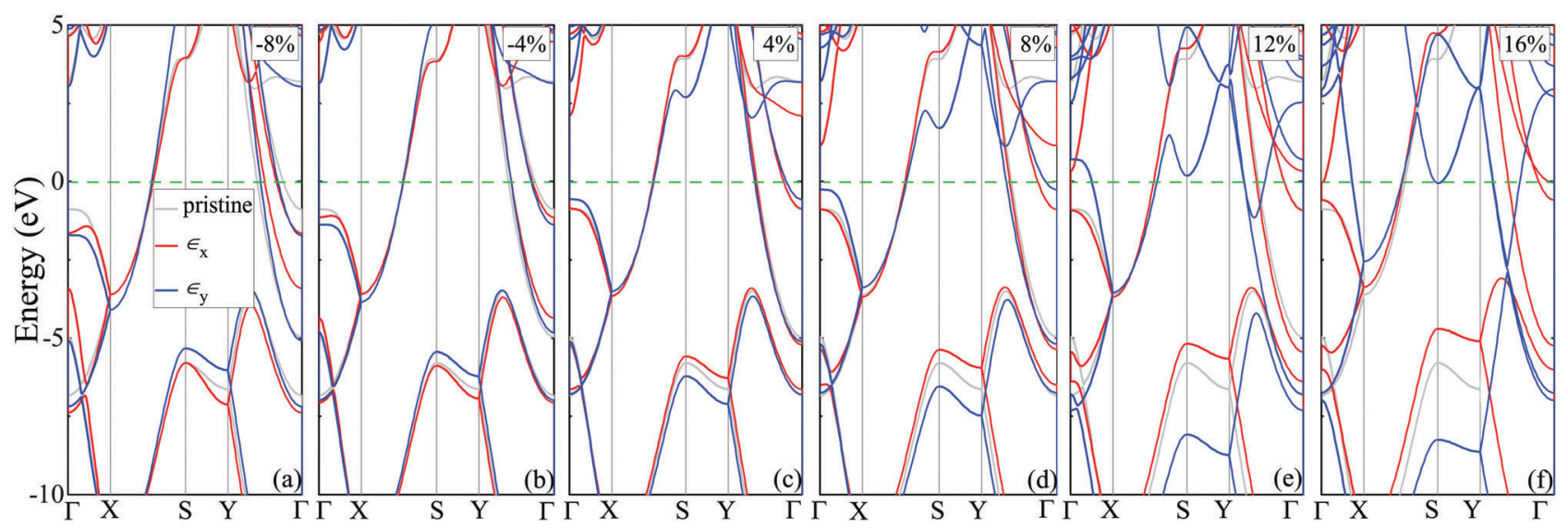

Fig. 2 The electronic band structures of borophene under (a) $-8 \%$, (b) $-4 \%$, (c) $4 \%$, (d) $8 \%$, (e) 12\%, and (f) $16 \%$ strain (compressive and tensile strain are indicated with negative and positive signs, respectively). The band diagrams along corrugated $\left(\epsilon_{x}\right)$ and uncorrugated $\left(\epsilon_{y}\right)$ strain directions are shown with red and blue colors, respectively. The band structure of the unstrained case is denoted by a gray solid line for comparison. The Fermi level is set to zero and is shown by a dashed green line.

the transverse one. All atoms are fully relaxed for each case. Subsequent to the geometry optimization, we calculate the strain-dependent electronic band structures of borophene as illustrated in Fig. 2. Even for high tensile/compressive strain levels, the metallicity of borophene is preserved. The effect of strain on the modification of the band structure is more pronounced along the uncorrugated direction, which is related to the buckled structure of borophene. This anisotropic response due to the applied strain can be associated with the significant discrepancy in in-plane stiffness along armchair (382 GPa nm) and zigzag axes (163 GPa nm). ${ }^{18,20}$ Moreover, the ultimate tensile strain regime along the armchair direction is $\sim 10 \%{ }^{20}$ where the irregularity in band curves is noticed. To gain a better understanding, we examine the variation of the buckling height $(\Delta h)$ with strain. $\Delta h$ decreases with increasing $\epsilon_{y}$, and the structure becomes completely planar at $\epsilon_{y}=16 \%$. On the other hand, $\epsilon_{x}$ has a minor effect on the buckling as shown in Fig. S1, ESI. $\dagger$ In order to quantify the modification of the band structure, we analyze the variation of d-BTE at high symmetry points under the influence of strain (see Table 1). d-BTE decreases with increasing tensile strain at $S$ and $\Gamma$ points for $\epsilon_{x}$ and $\epsilon_{y}$ up to $12 \%$, and it starts to increase beyond this strain level along the $y$ direction at the $S$ high-symmetry point where irregularities in band curves are present. On the other hand, band widths are seen to open-up further with increasing compressive strain at $S$ and $\Gamma$ points along both directions. Once a comparison between the compressive and tensile characters at identical strain amounts (i.e. $\pm 8 \%$ ) is made, it is observed that the band widths opened-up by around $2 \mathrm{eV}$ for one of the strain axes, both at $\Gamma$ and $S$ points; while different closing amounts of transition energies are found at $+8 \%$ tensile strain for $\Gamma$ and $S$ points. A common observation for both compressive and tensile strain is that the response to the applied strain in terms of the electronic properties is anisotropic, depending on the corrugated and uncorrugated directions in the lattice.

After obtaining the strain dependent electronic band structures, the frequency dependent imaginary dielectric function $\left[\varepsilon_{2}(\omega)\right]$ and optical conductivity $[\sigma(\omega)]$ of borophene are investigated. The in-plane optical responses for the cases when light is polarized along $x$ - and $y$-directions in the lattice under the effect of longitudinal tensile and compressive strain are plotted in terms of $\varepsilon_{2}^{x}(\omega)$ and $\varepsilon_{2}^{y}(\omega)$ in Fig. 3(a and b). Hereby, we only discuss the optical response of the structure along the direction where uniaxial strain is applied. The optical properties along the transverse direction are shown in Fig. S2, ESI. $\dagger$ Firstly, due to the metallic character of the borophene, a Drude peak appearing due to the free carrier intraband transitions in the low frequency (infrared) regime of $\varepsilon_{2}(\omega)$ (and also $\sigma(\omega)$ ) is included for both light polarization directions (see Fig. 3). This critical feature was usually not considered in previous studies. ${ }^{36}$ As the metallicity of borophene is preserved under strain, a Drude peak is obtained for all the strained cases. As seen in $\varepsilon_{2}^{x}(\omega)$ in Fig. 3(a), the main absorption peak of the unstrained borophene is located at $\sim 5.8 \mathrm{eV}$, lying in the UV range. ${ }^{53}$ While tensile strain red-shifts the interband absorption onsets, 

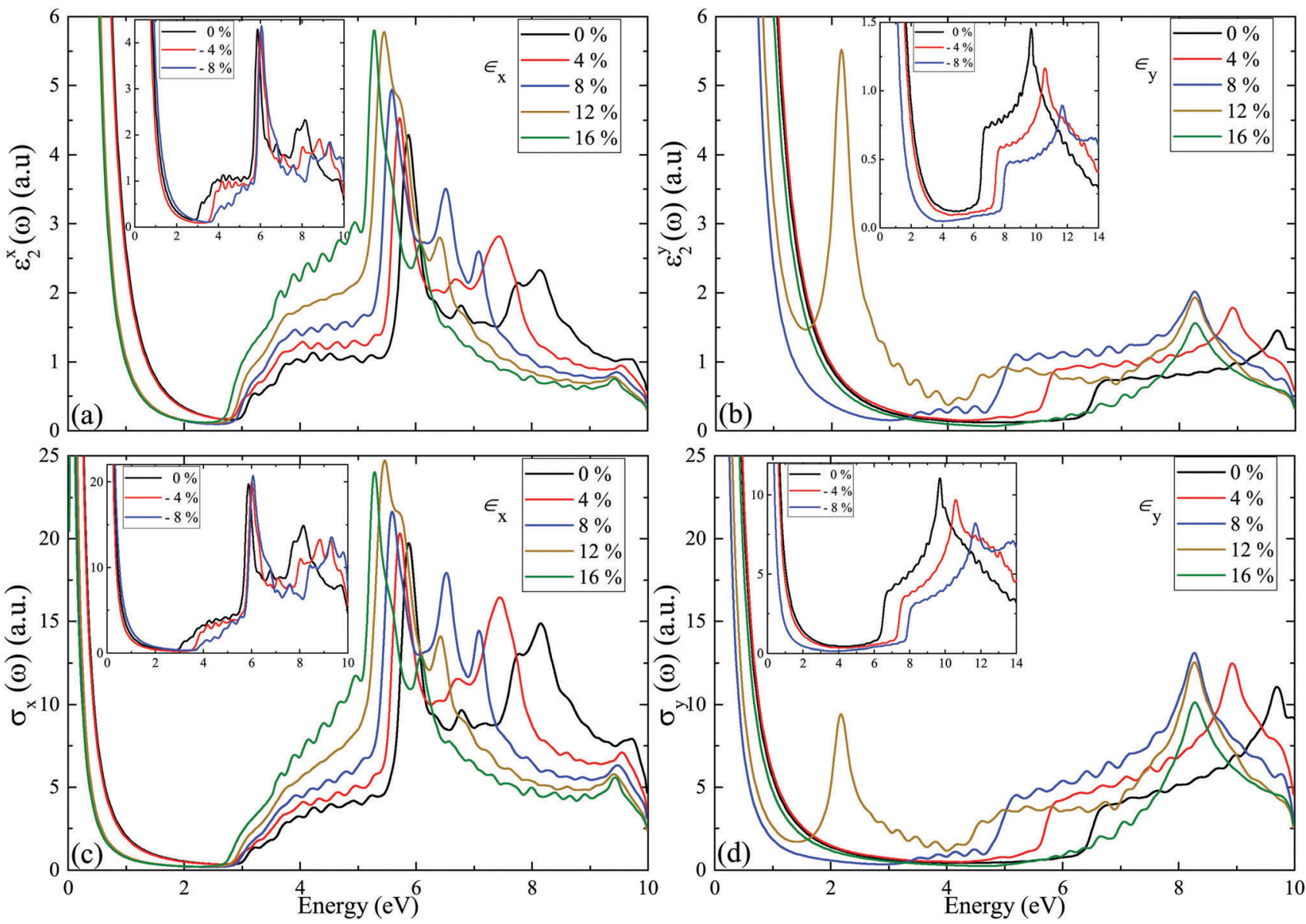

Fig. 3 Frequency dependent imaginary dielectric function (a) $\varepsilon_{2}^{x}(\omega)$ for light polarized along the $x$-direction, and (b) $\varepsilon_{2}^{y}(\omega)$ along the $y$-direction, and optical conductivity (c) $\sigma_{x}(\omega)$ along the $x$-direction, and (d) $\sigma_{y}(\omega)$ along the $y$-direction, for pristine and strained borophene.

absorption peaks and the overall spectra towards lower photon energies regularly for increasing amounts of strain, compressive strain blue-shifts the spectra which is illustrated as an inset of Fig. 3(a). Hence, regarding the in-plane optical response of monolayer unstrained and strained borophene along the $x$-direction, the absorption onset is set within/near the visible light regime (i.e., below $2.8 \mathrm{eV}$ for tensile and above $3.5 \mathrm{eV}$ for compressive), with light absorption extending beyond the visible range towards UV. In a similar manner, the variation of $\varepsilon_{2}^{y}(\omega)$ as a function of $\epsilon_{y}$ is shown in Fig. 3(b). The main absorption peak of pristine borophene is located at $\sim 9.7 \mathrm{eV}$ which corresponds to shorter wavelengths when compared with polarization along the $x$-direction. The tensile strain red-shifts $\varepsilon_{2}^{y}(\omega)$ up to $\epsilon_{y}=8 \%$, and the absorption band edge down until $\sim 4.5 \mathrm{eV}$, and next the main luminescence peaks remain fixed at $\sim 8.2 \mathrm{eV}$. Both major luminescence peaks (in $\varepsilon_{2}^{x}(\omega)$ and $\left.\varepsilon_{2}^{y}(\omega)\right)$ correspond to the prominent direct interband transitions at $\Gamma$ and $S$ points as listed in Table 1 . The anomalous absorption peak $(\sim 2.1 \mathrm{eV})$ at $\epsilon_{y}=12 \%$ is related to the structural irregularity discussed above. At this strain level, the buckled structure is found to be heavily deformed as presented in Fig. S1, ESI, $\dagger$ hence the onset of a structural transition (from buckled to planar) results in an unforeseen behavior of the optical response. Similar to polarization along the $x$-direction, the compressive strain blue-shifts the overall spectra, including the interband edge and luminescence peak positions. The variations within $\varepsilon_{2}^{y}(\omega)$ appear to be more significant than $\varepsilon_{2}^{x}(\omega)$, in compliance with the band widths discussed earlier, as the structure is more sensitive to strain along the armchair direction. Additionally, the red- (blue-) shifts of the absorption band edges and peak positions as a function of tensile (compressive) strain for both polarization directions are realized to be in compliance with the decreasing (increasing) d-BTE. It is also worth mentioning that the peak amplitudes of $\varepsilon_{2}^{x}(\omega)$ are significantly higher with peak ranges being much less dispersed than those of $\varepsilon_{2}^{y}(\omega)$.

Once $\varepsilon_{2}(\omega)$ is obtained, various spectral properties can further be calculated. Taking into account the metallic features of borophene, we examine $\sigma(\omega)$ for in-plane polarization of light. As evident in Fig. 3(c and d), $\sigma_{x}(\omega)$ and $\sigma_{y}(\omega)$ are damped to almost zero in the low energy regime beyond the Drude peak, indicating that the system is transparent in the infrared and visible regions. Further to the onset around $3 \mathrm{eV}, \sigma_{x}(\omega)$ of the unstrained structure displays an abrupt increase in absorption beyond $\sim 5.5 \mathrm{eV}$ in the $\mathrm{UV}$ region and a second peak at $\sim 8 \mathrm{eV} .^{35}$ Under the applied tensile (compressive) strain, the spectrum is 
globally red- (blue-) shifted with increasing amplitude. The interband transitions appear to be effective above $\sim 3 \mathrm{eV}$, until at least $9 \mathrm{eV}$ for all cases. On the other hand, $\sigma_{y}(\omega)$ of unstrained borophene shows no significant optical activity up to $\sim 6.5 \mathrm{eV}$, displaying a main peak at $\sim 9.7 \mathrm{eV}$ as shown in Fig. 3(c). In a similar manner, optical conductivity peaks are red- (blue-) shifted and peak amplitudes increase (decrease) for strain values up to $\pm 8 \%$. However, the amplitude of $\sigma_{y}(\omega)$ decreases for tensile strain values up to $16 \%$ while peak positions remained unchanged. The irregular peak feature at $12 \%$ can be associated with the onset of a structural transition from buckled to planar geometry as discussed above.

The optical response of borophene is calculated by the assumption of normal incident light, composed of in-plane and out-of plane components. The out-of-plane component (i.e. linearly polarized light perpendicular to the $c$-axis) of the imaginary dielectric function $\left(\varepsilon_{2}^{z}(\omega)\right)$ of borophene with and without the effect of strain is presented in Fig. S3, ESI. $\dagger$ The resulting red- and blue-shifts of the optical absorption spectra upon tensile or compressive strain are similar to the in-plane responses. No early Drude peaks are expected though, as the strong interband transitions for out-of-plane absorption come into play at significantly higher photon energies compared to the in-plane component, where for graphene and silicene a similar observation has been made. ${ }^{54,55}$

\section{B. The effect of functionalization}

Next, we examine the modification of the electronic and optical properties of borophene by surface functionalization. Hydrogenation $^{56}$ and halogenation ${ }^{57}$ has been used to tailor the intrinsic properties of $2 \mathrm{D}$ materials and even new derivatives can be obtained. For instance, hydrogenation of graphene (i.e. graphane) turns the semimetallic system into an insulator and transport properties also dramatically change..$^{58}$ Additionally, fluorographene is a good quality insulator with high thermal and chemical stability with optical and electrical properties being dramatically different both from bare and hydrogenated graphene.$^{57}$ In a similar manner, covering the surface of borophene with hydrogen and fluorine atoms radically modifies the properties of the pristine system and enhances the structural stability. ${ }^{19,35}$

Several phases of fully hydrogenated borophene have been predicted theoretically and the dynamic stability of these structures was tested with phonon dispersion analysis and molecular dynamics simulations. ${ }^{59-61}$ Recently, hydrogen boride sheets with an empirical formula of $\mathrm{B}_{1} \mathrm{H}_{1}$ were also realized by an exfoliation and ion-exchange method. ${ }^{38}$ The dynamically stable structure of the fully hydrogenated borophene (i.e. borophane; $\mathrm{BH})^{59-61}$ considered in this study is shown in Fig. 4(a). The optimized lattice constants $a$ and $b$ of BH are calculated as $2.83 \AA$ and $1.94 \AA$, respectively, which are compatible with previous findings. ${ }^{59,60}$ The electronic band structure of $\mathrm{BH}$ displayed in Fig. 4(a) indicates that $\mathrm{BH}$ has a semi-metallic character together with a Dirac cone at $E_{\mathrm{F}}$ between $Y$ and $\Gamma$ points. Different from graphene, the Dirac cone feature of BH's band structure is slightly distorted and asymmetric. ${ }^{60}$ Another important feature (a)
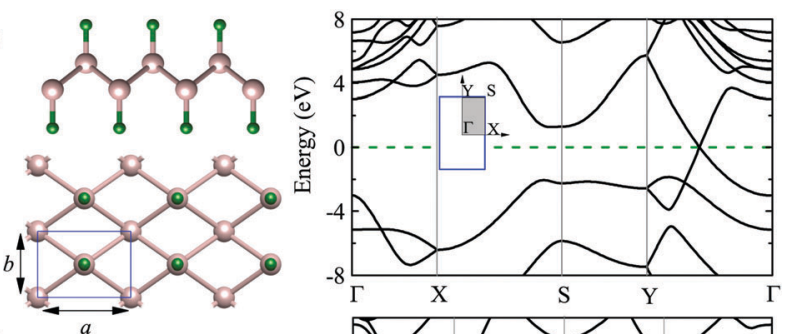

(b)
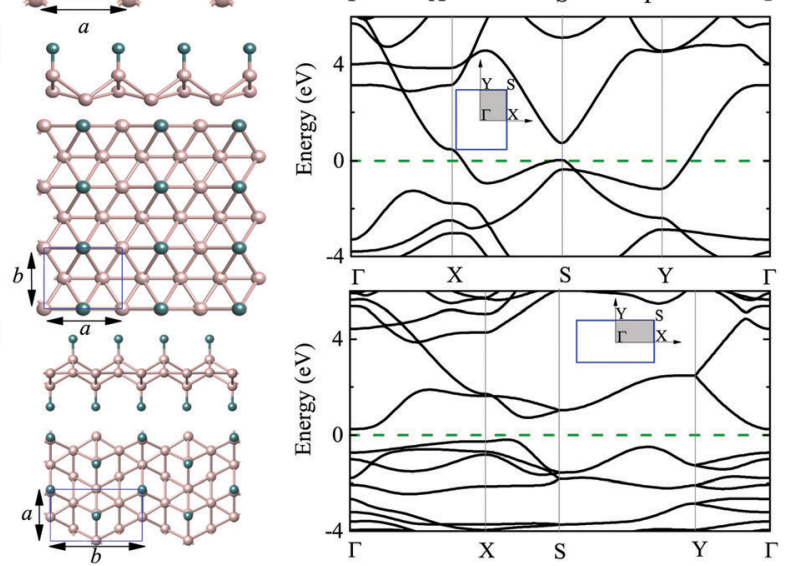

Fig. 4 Atomic configurations of (a) hydrogenated, $\mathrm{BH}$ and fluorinated, (b) $\mathrm{B}_{4} \mathrm{~F}$ and (c) $\mathrm{B}_{2} \mathrm{~F}$ systems along with their electronic band structures.

in BH's electronic properties is that d-BTE is observed as $\sim 6 \mathrm{eV}$ and $\sim 3 \mathrm{eV}$ at the $\Gamma$ and $S$ symmetry points, respectively.

Following its electronic properties, the optical properties of BH are investigated by calculating $\varepsilon_{2}(\omega)$ and $\sigma(\omega)$ as shown in Fig. 5(a) and (d). Similar to bare borophene, the optical response of BH is also anisotropic. Due to the graphene-like Dirac cone at $E_{\mathrm{F}}$, the Drude peak is obtained at the low energy regime for both polarization directions. The first absorption peaks of $\varepsilon_{2}^{x}(\omega)$ and $\varepsilon_{2}^{y}(\omega)$ are detected at $\sim 3.5 \mathrm{eV}$, however the amplitude and the energy range of the peak along $y$-polarization are significantly larger. When compared with pristine borophene, hydrogenation red-shifts the first absorption peaks but they still remain in the UV region. On the other hand, the second major peaks extend to the far UV region and are observed at $\sim 9 \mathrm{eV}$ with a larger amplitude along $x$-polarization. Likewise, in a previous study, borophene hydride was found to be metallic, ${ }^{37}$ yet the Drude peak was not shown in the optical spectrum, most probably since the intraband contributions were not taken into account in the calculation. The absorption edges of $\varepsilon_{2}^{x}(\omega)$ and $\varepsilon_{2}^{y}(\omega)$ are located in the visible region. When optical conductivity is examined, $\sigma(\omega)$ appears to be low but notable in the visible range for both polarization directions, different from the pristine system. The first major peaks of $\sigma_{x}(\omega)$ and $\sigma_{y}(\omega)$ are observed at $\sim 3.5 \mathrm{eV}$, with increasing amplitudes of the spectra towards higher photon energies.

Even though experimentally not yet realized, fluorinated borophene structures with varying coverage concentrations have been also foreseen. ${ }^{62,63}$ Interestingly, while fully fluorinated borophene (BF) was not found to be stable, single-sided coverage with $25 \%$ fluorination $\left(\mathrm{B}_{4} \mathrm{~F}\right)$ and double-sided coverage with $50 \%$ fluorination $\left(\mathrm{B}_{2} \mathrm{~F}\right)$ were shown to be dynamically stable. ${ }^{62}$ 

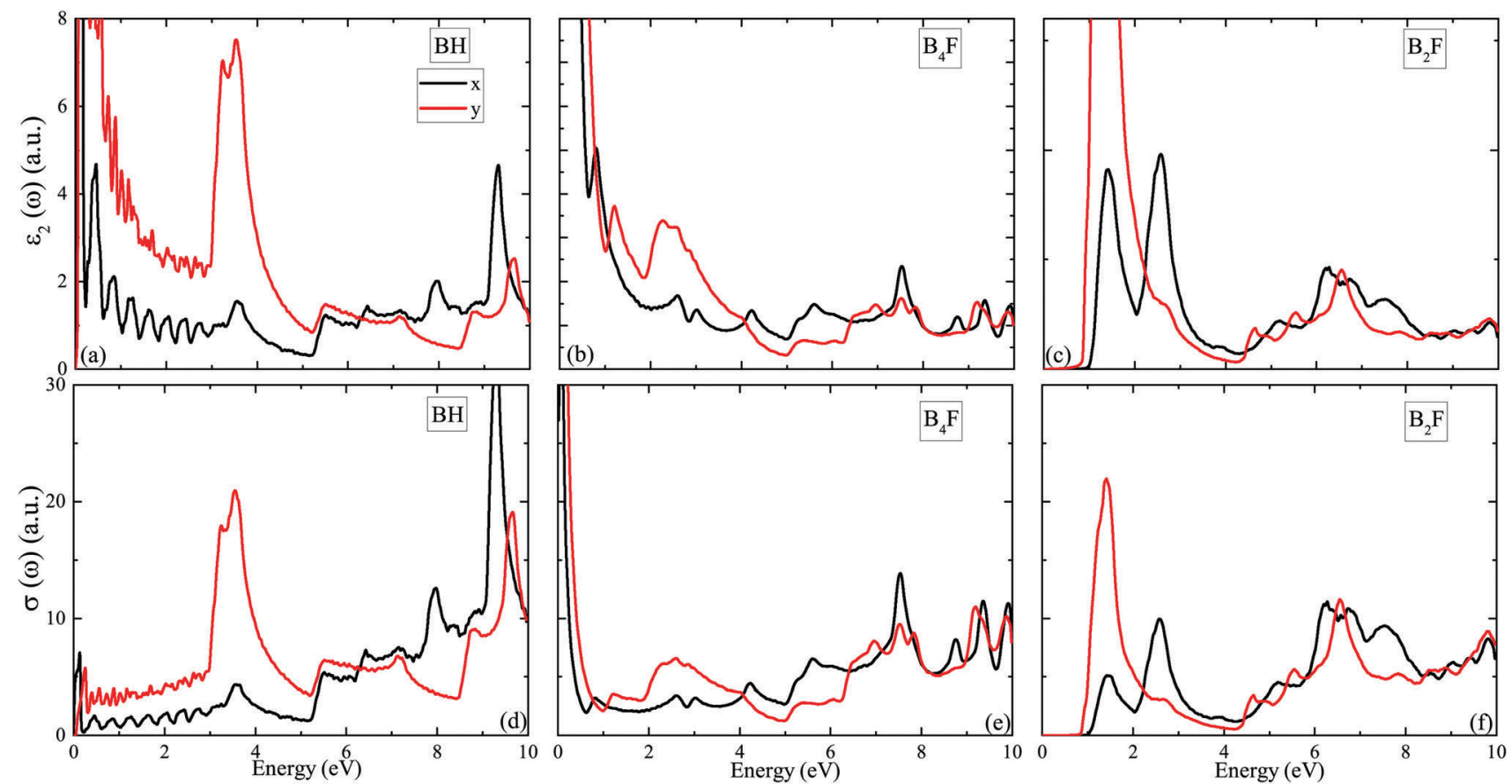

Fig. 5 Frequency dependent imaginary dielectric function $\left(\varepsilon_{2}(\omega)\right)$, and optical conductivity $(\sigma(\omega))$ of $\left(a\right.$ and d) $B H$, $\left(b\right.$ and e) $B_{4} F$, and $(c$ and $f) B_{2} F$ systems.

The structures of $\mathrm{B}_{4} \mathrm{~F}$ and $\mathrm{B}_{2} \mathrm{~F}$ which can be realized by using $2 \times 1$ and $2 \times 2$ super cells of pristine borophene, respectively, are shown in Fig. 4(b) and (c). The optimized lattice constants $a$ and $b$ of $\mathrm{B}_{4} \mathrm{~F}\left(\mathrm{~B}_{2} \mathrm{~F}\right)$ are calculated as $3.25 \AA(3.17 \AA)$ and $2.88 \AA$ (5.76 $\mathrm{A})$, respectively. While $\mathrm{B}_{4} \mathrm{~F}$ is metallic similar to pristine borophene, $\mathrm{B}_{2} \mathrm{~F}$ is found to be a narrow gap semiconductor with a $0.44 \mathrm{eV}$ indirect band gap. Prominent band width values for $\mathrm{B}_{4} \mathrm{~F}$ are given as $\sim 6, \sim 2$ and $\sim 6 \mathrm{eV}$ for the $\Gamma, X$ and $Y$ symmetry points, respectively.

Similar to the other metallic cases, $\mathrm{B}_{4} \mathrm{~F}$ displays significant Drude peaks in $\varepsilon_{2}(\omega)$ once the intraband transitions are included, while the band edges are observed at $\sim 0.6 \mathrm{eV}$ and $\sim 1.0 \mathrm{eV}$ for polarizations along the $x$ - and $y$-directions, respectively, if the intraband transitions are excluded. $\varepsilon_{2}(\omega)$ of $\mathrm{B}_{4} \mathrm{~F}$ also reveals two main absorption peaks at $\sim 2.5 \mathrm{eV}$ and $\sim 7.5 \mathrm{eV}$ for both light polarization directions. These peaks appear at lower energies compared to $\mathrm{BH}$, the first one remaining in the visible range. It should also be mentioned that the anisotropy of the optical response is less evident compared to other structures considered in this study. On the other hand, the semiconductor phase, $\mathrm{B}_{2} \mathrm{~F}$ has clear absorption onsets at $\sim 1 \mathrm{eV}$ and $\sim 0.7 \mathrm{eV}$ in $\varepsilon_{2}^{x}(\omega)$ and $\varepsilon_{2}^{y}(\omega)$, respectively, in compliance with the direct band gap of $\sim 0.99 \mathrm{eV}$ at $\Gamma$. While $\mathrm{B}_{2} \mathrm{~F}$ has two successive absorption peaks along $x$-polarization, it displays a single remarkable peak for $y$-polarization. Hence, $\varepsilon_{2}(\omega)$ yielding prominent luminescence peaks also in the visible range, indicates that visible light can be absorbed. While $\sigma(\omega)$ is pronounced throughout the whole spectral range for $\mathrm{B}_{4} \mathrm{~F}$, for $\mathrm{B}_{2} \mathrm{~F}$ it is rather discontinuous with peaks mainly concentrated around $\sim 0.7-3.0$ and $\sim 7 \mathrm{eV}$. Our results also suggest that in addition to fluorine, functionalization of borophene with other halogen atoms (i.e., $\mathrm{Cl}$ ) can lead to new derivatives with interesting optoelectonic properties.
The out-of-plane components of the imaginary dielectric functions $\left(\varepsilon_{2}^{z}(\omega)\right)$ of $\mathrm{BH}, \mathrm{B}_{2} \mathrm{~F}$ and $\mathrm{B}_{4} \mathrm{~F}$ systems, with and without the effect of strain, are shown in Fig. S4, ESI. $\dagger$ Accordingly, the onsets of out-of-plane absorption are located beyond $\sim 2,5$ and $8 \mathrm{eV}$, for $\mathrm{B}_{2} \mathrm{~F}, \mathrm{~B}_{4} \mathrm{~F}$ and $\mathrm{BH}$ systems, respectively. Prominent and distinct peaks are observed for $\mathrm{BH}$ and $\mathrm{B}_{4} \mathrm{~F}$, slightly above $8 \mathrm{eV}$ for the former and below $10 \mathrm{eV}$ for the latter, located beyond the far UV region.

\section{Conclusion}

In summary, we investigate the modification of the electronic and optical properties of borophene by applied strain and surface functionalization. The metallicity of borophene is preserved even at high strain levels where strong structural deformations are observed. Besides, the applied load tunes the optical response which is correlated with the structural changes (i.e. buckling height) and alteration of band transition energies. The tensile (compressive) strain red-(blue-) shifts the main absorption peaks, nevertheless the first absorption peaks still remain in the UV range and a Drude peak is obtained for each case due to intraband transitions. The effect of strain is more pronounced along the uncorrugated direction in the lattice, indicating an anisotropic response of borophene to both the light polarization and the direction of the applied load. The calculated optical conductivity remains very small in the visible regime for the strain range covered. On the other hand, surface functionalization dramatically modifies both the electronic and optical properties. The coverage of borophene with hydrogen makes the system a semi-metal with a Dirac cone and first absorption peaks shift to the near UV range, beyond the 
visible-light edge. Upon fluorination of borophene, the system remains metallic for $25 \%$ coverage $\left(\mathrm{B}_{4} \mathrm{~F}\right)$, however becomes a semiconductor at $50 \%\left(\mathrm{~B}_{2} \mathrm{~F}\right)$. The first absorption peaks are obtained in the infrared and visible frequencies, hence these systems can absorb visible light. Differently from the pristine case, functionalized borophene displays significant optical conductivity in the visible regime. Our results demonstrate the tunability of the optoelectronic properties of borophone, which is expected to facilitate various potential applications.

\section{Conflicts of interest}

There are no conflicts to declare.

\section{Acknowledgements}

The calculations were performed at TUBITAK ULAKBIM, High Performance and Grid Computing Center (TR-Grid e-Infrastructure) and the National Center for High Performance Computing of Turkey (UHeM) under grant no. 5003622015. This work was supported by the Scientific and Technological Research Council of Turkey (TUBITAK) under Project No. 115F088. E. D. acknowledges support from The Turkish Academy of Sciences - Outstanding Young Scientists Award Program (TUBA-GEBIP). A. M. and Y. M. acknowledge the Ankara University for high performance computing facility thorough the AYP under Grand No. 17A0443001.

\section{References}

1 F. Xia, H. Wang, D. Xiao, M. Dubey and A. Ramasubramaniam, Nat. Photonics, 2014, 8, 899.

2 H. Zhao, Q. Guo, F. Xia and H. Wang, Nanophotonics, 2015, 4, 128.

3 M. Liu, X. Yin, E. Ulin-Avila, B. Geng, T. Zentgraf, L. Ju, F. Wang and X. Zhang, Nature, 2011, 474, 64.

4 A. Pospischil, M. Humer, M. M. Furchi, D. Bachmann, R. Guider, T. Fromherz and T. Mueller, Nat. Photonics, 2013, 7, 892.

5 G. Eda and S. A. Maier, ACS Nano, 2013, 7, 5660.

6 A. K. Geim and K. S. Novoselov, Nat. Mater., 2007, 6, 183.

7 K. F. Mak, L. Ju, F. Wang and T. F. Heinz, Solid State Commun., 2012, 152, 1341.

8 F. Xia, H. Yan and P. Avouris, Proc. IEEE, 2013, 101, 1717.

9 F. Xia, T. Mueller, Y.-m. Lin, A. Valdes-Garcia and P. Avouris, Nat. Nanotechnol., 2009, 4, 839.

10 O. D. Miller, O. Ilic, T. Christensen, M. H. Reid, H. A. Atwater, J. D. Joannopoulos, M. Soljacic and S. G. Johnson, Nano Lett., 2017, 17, 5408.

11 K. F. Mak, C. Lee, J. Hone, J. Shan and T. F. Heinz, Phys. Rev. Lett., 2010, 105, 136805.

12 A. Splendiani, L. Sun, Y. Zhang, T. Li, J. Kim, C.-Y. Chim, G. Galli and F. Wang, Nano Lett., 2010, 10, 1271.

13 O. Lopez-Sanchez, D. Lembke, M. Kayci, A. Radenovic and A. Kis, Nat. Nanotechnol., 2013, 8, 497.
14 M. M. Ugeda, A. J. Bradley, S.-F. Shi, H. Felipe, Y. Zhang, D. Y. Qiu, W. Ruan, S.-K. Mo, Z. Hussain and Z.-X. Shen, et al., Nat. Mater., 2014, 13, 1091.

15 F. Xia, H. Wang and Y. Jia, Nat. Commun., 2014, 5, 4458.

16 M. Buscema, D. J. Groenendijk, S. I. Blanter, G. A. Steele, H. S. Van Der Zant and A. Castellanos-Gomez, Nano Lett., 2014, 14, 3347.

17 V. Tran, R. Soklaski, Y. Liang and L. Yang, Phys. Rev. B: Condens. Matter Mater. Phys., 2014, 89, 235319.

18 A. J. Mannix, X.-F. Zhou, B. Kiraly, J. D. Wood, D. Alducin, B. D. Myers, X. Liu, B. L. Fisher, U. Santiago and J. R. Guest, et al., Science, 2015, 350, 1513.

19 B. Peng, H. Zhang, H. Shao, Y. Xu, R. Zhang and H. Zhu, J. Mater. Chem. C, 2016, 4, 3592.

20 B. Mortazavi, O. Rahaman, A. Dianat and T. Rabczuk, Phys. Chem. Chem. Phys., 2016, 18, 27405.

21 H. Sun, Q. Li and X. Wan, Phys. Chem. Chem. Phys., 2016, 18, 14927.

22 H. Xiao, W. Cao, T. Ouyang, S. Guo, C. He and J. Zhong, Sci. Rep., 2017, 7, 45986.

23 B. Mortazavi, M.-Q. Le, T. Rabczuk and L. F. C. Pereira, Phys. E, 2017, 93, 202.

24 E. S. Penev, A. Kutana and B. I. Yakobson, Nano Lett., 2016, 16, 2522.

25 M. Gao, Q.-Z. Li, X.-W. Yan and J. Wang, Phys. Rev. B, 2017, 95, 024505.

26 R. Xiao, D. Shao, W. Lu, H. Lv, J. Li and Y. Sun, Appl. Phys. Lett., 2016, 109, 122604.

27 B. Feng, J. Zhang, Q. Zhong, W. Li, S. Li, H. Li, P. Cheng, S. Meng, L. Chen and K. Wu, Nat. Chem., 2016, 8, 563.

28 B. Feng, O. Sugino, R.-Y. Liu, J. Zhang, R. Yukawa, M. Kawamura, T. Iimori, H. Kim, Y. Hasegawa and H. Li, et al., Phys. Rev. Lett., 2017, 118, 096401.

29 X. Wu, J. Dai, Y. Zhao, Z. Zhuo, J. Yang and X. C. Zeng, ACS Nano, 2012, 6, 7443.

30 E. S. Penev, S. Bhowmick, A. Sadrzadeh and B. I. Yakobson, Nano Lett., 2012, 12, 2441.

31 X.-F. Zhou, X. Dong, A. R. Oganov, Q. Zhu, Y. Tian and H.-T. Wang, Phys. Rev. Lett., 2014, 112, 085502.

32 S. H. Mir, S. Chakraborty, P. C. Jha, J. Wärnå, H. Soni, P. K. Jha and R. Ahuja, Appl. Phys. Lett., 2016, 109, 053903.

33 H. Jiang, Z. Lu, M. Wu, F. Ciucci and T. Zhao, Nano Energy, 2016, 23, 97.

34 J. Wang, Y. Du and L. Sun, Int. J. Hydrogen Energy, 2016, 41, 5276.

35 A. Lherbier, A. R. Botello-Méndez and J.-C. Charlier, $2 D$ Mater., 2016, 3, 045006.

36 L. Adamska and S. Sharifzadeh, ACS Omega, 2017, 2, 8290.

37 B. Mortazavi, M. Makaremi, M. Shahrokhi, M. Raeisi, C. V. Singh, T. Rabczuk and L. F. C. Pereira, Nanoscale, 2018, 10, 3759.

38 H. Nishino, T. Fujita, N. T. Cuong, S. Tominaka, M. Miyauchi, S. Iimura, A. Hirata, N. Umezawa, S. Okada and E. Nishibori, et al., J. Am. Chem. Soc., 2017, 139, 13761.

39 Y. Jiao, F. Ma, J. Bell, A. Bilic and A. Du, Angew. Chem., 2016, 128, 10448. 
40 W. Kohn and L. J. Sham, Phys. Rev., 1965, 140, A1133.

41 P. Hohenberg and W. Kohn, Phys. Rev., 1964, 136, B864.

42 G. Kresse and J. Hafner, Phys. Rev. B: Condens. Matter Mater. Phys., 1993, 47, 558.

43 G. Kresse and J. Hafner, Phys. Rev. B: Condens. Matter Mater. Phys., 1994, 49, 14251.

44 G. Kresse and J. Furthmüller, Comput. Mater. Sci., 1996, $6,15$.

45 G. Kresse and J. Furthmüller, Phys. Rev. B: Condens. Matter Mater. Phys., 1996, 54, 11169.

46 M. Gajdoš, K. Hummer, G. Kresse, J. Furthmüller and F. Bechstedt, Phys. Rev. B: Condens. Matter Mater. Phys., 2006, 73, 045112.

47 J. P. Perdew, K. Burke and M. Ernzerhof, Phys. Rev. Lett., 1996, 77, 3865.

48 J. P. Perdew, K. Burke and M. Ernzerhof, Phys. Rev. Lett., 1997, 78, 1396.

49 H. J. Monkhorst and J. D. Pack, Phys. Rev. B: Solid State, 1976, 13, 5188.

50 D. Bohm and D. Pines, Phys. Rev., 1951, 82, 625.

51 D. Pines and D. Bohm, Phys. Rev., 1952, 85, 338.

52 D. Bohm and D. Pines, Phys. Rev., 1953, 92, 609.

53 It should be noted that while main peak is found at $\sim 5.8 \mathrm{eV}$ by DFT-PBE, the same peak is obtained at $\sim 6.3 \mathrm{eV}$ when hybrid functionals (HSE) are used. This indicates a possible blue-shift of the spectrum by $\sim 0.7 \mathrm{eV}$ resulting from the underestimated band widths by DFT-PBE.

54 L. Matthes, O. Pulci and F. Bechstedt, Phys. Rev. B, 2016, 94, 205408.

55 W. Hu, Z. Li and J. Yang, J. Chem. Phys., 2013, 139, 154704.

56 M. Pumera and C. H. A. Wong, Chem. Soc. Rev., 2013, 42, 5987.

57 R. R. Nair, W. Ren, R. Jalil, I. Riaz, V. G. Kravets, L. Britnell, P. Blake, F. Schedin, A. S. Mayorov and S. Yuan, et al., Small, 2010, 6, 2877.

58 D. C. Elias, R. R. Nair, T. Mohiuddin, S. Morozov, P. Blake, M. Halsall, A. Ferrari, D. Boukhvalov, M. Katsnelson and A. Geim, et al., Science, 2009, 323, 610.

59 N. K. Jena, R. B. Araujo, V. Shukla and R. Ahuja, ACS Appl. Mater. Interfaces, 2017, 9, 16148.

60 L.-C. Xu, A. Du and L. Kou, Phys. Chem. Chem. Phys., 2016, 18, 27284.

61 Z.-Q. Wang, T.-Y. Lu, H.-Q. Wang, Y. P. Feng and J.-C. Zheng, RSC Adv., 2017, 7, 47746.

62 R. Pekoz, M. Konuk, M. E. Kilic and E. Durgun, ACS Omega, 2018, 3, 1815.

63 J. Khanifaev, R. Peköz, M. Konuk and E. Durgun, Phys. Chem. Chem. Phys., 2017, 19, 28963. 